\title{
Norm inequalities for submultiplicative functions involving contraction sector $2 \times 2$ block matrices
}

Xiaoying Zhou ${ }^{1,2,3^{*}}$

"Correspondence:
zhouxy213@163.com
'Department of Mathematics and
Statistics, Hainan Normal University,
Haikou, China
${ }^{2}$ Key Laboratory of Data Science
and Intelligence Education Ministry
of Education, Hainan Normal
University, Haikou, China
Full list of author information is
available at the end of the article

\begin{abstract}
In this article, we show unitarily invariant norm inequalities for sector $2 \times 2$ block matrices which extend and refine some recent results of Bourahli, Hirzallah, and Kittaneh (Positivity, 2020, https://doi.org/10.1007/s11117-020-00770-w).
\end{abstract}

MSC: $15 \mathrm{~A} 18 ; 15 \mathrm{~A} 45$

Keywords: Contraction sector block matrices; Unitarily invariant norms; Submultiplicative functions

\section{Introduction}

Let $\mathbb{M}_{n}$ be a set of all $n \times n$ complex matrices. A matrix $A \in \mathbb{M}_{n}$ is said to be positive semidefinite if $x^{*} A x \geq 0$ for all $x \in \mathbb{C}^{n}$. If the eigenvalues $\lambda_{1}(A), \ldots, \lambda_{n}(A)$ of $A$ are all real, we arrange them in nonincreasing order $\lambda_{1}(A) \geq \cdots \geq \lambda_{n}(A)$. Singular values of $A$ are the eigenvalues of $|A|$ and are arranged in nonincreasing order $s_{1}(A) \geq \cdots \geq s_{n}(A)$. For $A \in \mathbb{M}_{n}$, we denote by $|A|=\left(A^{*} A\right)^{\frac{1}{2}}, A^{*},\|A\|$, and $\|A\|_{\infty}=s_{1}(A)$ the absolute value, the conjugate transpose, the unitarily invariant norm, and the operator norm, respectively. We say $A$ is a contraction if $\|A\|_{\infty} \leq 1$. By convention, the $n \times n$ identity matrix is denoted by $I_{n} \cdot\|A\|$ and $\|A\|_{\infty}=s_{1}(A)$ are unitarily invariant, i.e., $\|U A V\|=\|A\|$ for all unitary matrices $U, V$. For $A, B \in \mathbb{M}_{n}$, the weak majorization relation $s(A) \prec_{w} s(B)$ means

$$
\sum_{j=1}^{k} s_{j}(A) \leq \sum_{j=1}^{k} s_{j}(B), \quad k=1,2, \ldots, n .
$$

For $A \in \mathbb{M}_{n}$, recall the Cartesian (or Toeplitz) decomposition (see, e.g., [2, p. 6] and [3, p. 7])

$$
A=\operatorname{Re} A+i \operatorname{Im} A,
$$

where

$$
\operatorname{Re} A:=\frac{1}{2}\left(A+A^{*}\right), \quad \operatorname{Im} A:=\frac{1}{2 i}\left(A-A^{*}\right) .
$$

(c) The Author(s) 2020. This article is licensed under a Creative Commons Attribution 4.0 International License, which permits use, sharing, adaptation, distribution and reproduction in any medium or format, as long as you give appropriate credit to the original author(s) and the source, provide a link to the Creative Commons licence, and indicate if changes were made. The images or other third party material in this article are included in the article's Creative Commons licence, unless indicated otherwise in a credit line to the material. If material is not included in the article's Creative Commons licence and your intended use is not permitted by statutory regulation or exceeds the permitted use, you will need to obtain permission directly from the copyright holder. To view a copy of this licence, visit http://creativecommons.org/licenses/by/4.0/. 
The Cartesian decomposition of a matrix is unique. There are many interesting properties for such a decomposition. A celebrated result due to Fan and Hoffman (see, e.g., [2, p.73]) states that

$$
\lambda_{j}(\operatorname{Re} A) \leq s_{j}(A), \quad j=1, \ldots, n
$$

The numerical range of $A \in \mathbb{M}_{n}$ is defined by

$$
W(A)=\left\{x^{*} A x \mid x \in \mathbb{C}^{n}, x^{*} x=1\right\},
$$

which is a compact convex set (see, e.g., [4, Chap. 1]). For $\alpha \in[0, \pi / 2)$, a sector on the complex plane is

$$
S_{\alpha}=\{z \in \mathbb{C}|\operatorname{Re} z \geq 0,| \operatorname{Im} z \mid \leq(\operatorname{Re} z) \tan \alpha\} .
$$

A sector matrix $A \in \mathbb{M}_{n}$ is a matrix whose numerical range is contained in $S_{\alpha}$ for some $\alpha \in[0, \pi / 2)$. It is clear that if $A \in \mathbb{M}_{n}$ is a sector matrix, then $\operatorname{Re} A$ is positive semidefinite. The interested readers can refer to [5-10], and [4] for recent results on sector matrices. If $W(A)$ is contained in the first quadrant of the complex plane, then $\operatorname{Re} A$ and $\operatorname{Im} A$ are positive semidefinite. We call such a matrix $A$ accretive-dissipative. Note that if $A$ is accretive-dissipative, then $W\left(e^{-\frac{i \pi}{4}} A\right) \subseteq S_{\frac{\pi}{4}}$. Recently this class of matrices has been studied by researchers partly due to the fact that it contains the class of positive semidefinite matrices (see, e.g., [1, 11-17]).

Next we introduce a special class of functions. Let $\mathcal{C}$ be the class of all nonnegative increasing functions $f$ on $[0, \infty)$ preserving the weak-log majorization, i.e., for two nonincreasing sequences of nonnegative real numbers $\left(x_{1}, x_{2}, \ldots, x_{n}\right)$ and $\left(y_{1}, y_{2}, \ldots, y_{n}\right)$, $\prod_{j=1}^{k} x_{j} \leq \prod_{j=1}^{k} y_{j}$ for $k=1, \ldots, n$ implies $\prod_{j=1}^{k} f\left(x_{j}\right) \leq \prod_{j=1}^{k} f\left(y_{j}\right)$ for $k=1, \ldots, n$. There are many other properties on this class of functions; see $[12,18]$. A nonnegative function $f \in \mathcal{C}$ on the interval $[0, \infty)$ is said to be submultiplicative if $f(a b) \leq f(a) f(b)$ whenever $a, b \in[0, \infty)$. Recently, some unitarily invariant norm inequalities for submultiplicative functions of accretive-dissipative matrices have been shown in [12] and [13].

Bourahli et al. [1, Lemma 3.4] showed that if $A=\left(\begin{array}{ll}A_{11} & A_{12} \\ A_{21} & A_{22}\end{array}\right) \in \mathbb{M}_{2 n}$ is a positive semidefinite contraction and $s, t$ are positive real numbers such that $\frac{1}{s}+\frac{1}{t}=1$, then

$$
\left\|f\left(\left|A_{12}\right|^{2}\right)\right\| \leq\left\|f^{s}\left(A_{11}^{\frac{1}{2}}\right)\right\|^{\frac{1}{s}}\left\|f^{t}\left(A_{22}^{\frac{1}{2}}\right)\right\|^{\frac{1}{t}},
$$

where $f$ is an increasing submultiplicative function on $[0, \infty)$ with $f(0)=0$. Moreover, if $A \in \mathbb{M}_{2 n}$ is just positive semidefinite (not necessarily contraction matrices), then they presented a result related to (2) in [1, Remark 3.5] as follows:

$$
\left\|f\left(\left|A_{12}\right|^{2}\right)\right\| \leq f\left(\left\|A_{11}^{\frac{1}{2}}\right\|_{\infty}\left\|A_{22}^{\frac{1}{2}}\right\|_{\infty}\right)\left\|f^{s}\left(A_{11}^{\frac{1}{2}}\right)\right\|^{\frac{1}{s}}\left\|f^{t}\left(A_{22}^{\frac{1}{2}}\right)\right\|^{\frac{1}{t}} .
$$

\section{Unitarily invariant norms for submultiplicative functions}

In [12] and [13], some unitarily invariant norms for accretive-dissipative matrices involving a special class of functions have been shown. In this section, we present inequalities for sector block matrices involving the class of function. 
Lemma 2.1 ([19, p. 280]) Let $A, X, B$ be $m \times p, p \times q, q \times n$ matrices, respectively. Then

$$
s_{i}(A X B) \leq s_{1}(A) s_{j}(X) s_{1}(B), \quad i \leq \min \{m, p, q, n\} .
$$

Lemma $2.2\left(\left[8\right.\right.$, Theorem 2.1]) Let $A \in \mathbb{M}_{n}$ be $n \times n$ such that $W(A) \subseteq S_{\alpha}$ for some $\alpha \in$ $[0, \pi / 2)$. Then there exist an invertible matrix $X$ and a unitary and diagonal matrix $Z=$ $\operatorname{diag}\left(e^{i \theta_{1}}, \ldots, e^{i \theta_{n}}\right)$ with all $\left|\theta_{j}\right| \leq \alpha$ such that $A=X Z X^{*}$. Moreover, such a matrix $Z$ is unique up to permutation.

Lemma 2.3 ([8, Corollary 2.3 (ii)]) Let $A \in \mathbb{M}_{n}$ be such that $W(A) \subseteq S_{\alpha}$ for some $\alpha \in$ $[0, \pi / 2)$, and let $A=X Z X^{*}$ be a sectoral decomposition of $A$, where $X$ is invertible and $Z$ is unitary and diagonal. Then

$$
R R^{*} \leq \sec (\alpha)\left(R(\operatorname{Re} Z) R^{*}\right)=\sec (\alpha)\left(\operatorname{Re}\left(R Z R^{*}\right)\right)
$$

for every matrix $R \in \mathbb{M}_{n}$.

We are ready to present our main result of this section.

Theorem 2.4 Let $f \in \mathcal{C}$ be an increasing submultiplicative function on $[0, \infty)$ and $A \in \mathbb{M}_{2 n}$ be a contraction matrix partitioned as

$$
A=\left(\begin{array}{ll}
A_{11} & A_{12} \\
A_{21} & A_{22}
\end{array}\right)
$$

with $W(A) \subseteq S_{\alpha}$ for some $\alpha \in[0, \pi / 2)$. Then, for all $r, s, t>0$ with $\frac{1}{s}+\frac{1}{t}=1$ and all unitarily invariant norms,

$$
\left\|f\left(\left|A_{12}\right|^{2 r}\right)\right\| \leq\left\|f^{s}\left(\left(\sec ^{2}(\alpha)\left|A_{11}\right|\right)^{r / 2}\right)\right\|^{1 / s}\left\|f^{t}\left(\left(\sec ^{2}(\alpha)\left|A_{22}\right|\right)^{r / 2}\right)\right\|^{1 / t}
$$

and

$$
\left\|f\left(\left|A_{21}\right|^{2 r}\right)\right\| \leq\left\|f^{s}\left(\left(\sec ^{2}(\alpha)\left|A_{11}\right|\right)^{r / 2}\right)\right\|^{1 / s}\left\|f^{t}\left(\left(\sec ^{2}(\alpha)\left|A_{22}\right|\right)^{r / 2}\right)\right\|^{1 / t}
$$

Proof Note that $A$ is a sector matrix with $W(A) \subseteq S_{\alpha}$. By Lemma 2.2, we have $A=X Z X^{*}$, where $X$ is invertible and $Z$ is unitary and diagonal. We partition $X$ as $\left(\begin{array}{l}X_{1} \\ X_{2}\end{array}\right), X_{1}, X_{2} \in \mathbb{M}_{n \times 2 n}$. Thus, $\operatorname{Re} A_{11}=X_{1}(\operatorname{Re} Z) X_{1}^{*}, \operatorname{Re} A_{22}=X_{2}(\operatorname{Re} Z) X_{2}^{*}$, and $A_{12}=X_{1} Z X_{2}^{*}$. Consider the Cartesian decomposition

$$
A=\left(\begin{array}{ll}
A_{11} & A_{12} \\
A_{21} & A_{22}
\end{array}\right)=R+i S=\left(\begin{array}{ll}
R_{11} & R_{12} \\
R_{21} & R_{22}
\end{array}\right)+i\left(\begin{array}{ll}
S_{11} & S_{12} \\
S_{21} & S_{22}
\end{array}\right),
$$

where $R$ is positive semidefinite and $S$ is Hermitian. Since $A$ is a contraction matrix,

$$
A A^{*}=R^{2}+S^{2}+i(S R-R S) \leq I
$$


and

$$
A^{*} A=R^{2}+S^{2}+i(R S-S R) \leq I .
$$

Adding (7) and (8), we get

$$
2\left(R^{2}+S^{2}\right) \leq 2 I
$$

Thus, $R$ and $S$ are also contraction matrices, which implies that both $\operatorname{Re} A_{11}=R_{11}$ and $\operatorname{Re} A_{22}=R_{22}$ are positive semidefinite contractions.

Now

$$
\begin{aligned}
s_{\ell}\left(\left|A_{12}\right|^{r}\right) & =s_{\ell}^{r}\left(\left|A_{12}\right|\right)=s_{\ell}^{r}\left(A_{12}\right)=s_{\ell}^{r}\left(X_{1} Z X_{2}^{*}\right) \\
& \leq s_{1}^{r}\left(X_{1}\right) s_{\ell}^{r}\left(Z X_{2}^{*}\right) \quad(\text { by Lemma 2.1) } \\
& =\lambda_{1}^{\frac{r}{2}}\left(X_{1}^{*} X_{1}\right) \lambda_{\ell}^{\frac{r}{2}}\left(X_{2} Z^{*} Z X_{2}^{*}\right) \\
& =\lambda_{1}^{\frac{r}{2}}\left(X_{1} X_{1}^{*}\right) \lambda_{\ell}^{\frac{r}{2}}\left(X_{2} X_{2}^{*}\right) \\
& \leq \lambda_{1}^{\frac{r}{2}}\left(\sec (\alpha) X_{1}(\operatorname{Re} Z) X_{1}^{*}\right) \lambda_{\ell}^{\frac{r}{2}}\left(\sec (\alpha) X_{2}(\operatorname{Re} Z) X_{2}^{*}\right) \quad \text { (by Lemma 2.3) } \\
& =\lambda_{1}^{\frac{r}{2}}\left(\sec (\alpha) \operatorname{Re} A_{11}\right) \lambda_{\ell}^{\frac{r}{2}}\left(\sec (\alpha) \operatorname{Re} A_{22}\right) \\
& \leq \sec ^{\frac{r}{2}}(\alpha) \lambda_{\ell}^{\frac{r}{2}}\left(\sec (\alpha) \operatorname{Re} A_{22}\right) \quad\left(\operatorname{since} \operatorname{Re} A_{11}\right. \text { is a contraction) } \\
& \leq \sec ^{r}(\alpha) s_{\ell}^{\frac{r}{2}}\left(\left|A_{22}\right|\right) \quad(\text { by }(1))
\end{aligned}
$$

for $l=1,2, \ldots, n$.

Since $\operatorname{Re} A_{22}$ is also a contraction, it follows from (1) and (9) that

$$
s_{\ell}\left(\left|A_{12}\right|^{r}\right) \leq \sec ^{r}(\alpha) s_{\ell}^{\frac{r}{2}}\left(\left|A_{11}\right|\right)
$$

for $l=1,2, \ldots, n$.

Multiplying inequalities (10) and (11) by each other implies that

$$
s_{\ell}\left(\left|A_{12}\right|^{2 r}\right) \leq \sec ^{2 r}(\alpha) s_{\ell}^{\frac{r}{2}}\left(\left|A_{11}\right|\right) s_{\ell}^{\frac{r}{2}}\left(\left|A_{22}\right|\right)
$$

for $l=1,2, \ldots, n$.

So,

$$
\begin{aligned}
s_{\ell}\left(f\left(\left|A_{12}\right|^{2 r}\right)\right)= & f\left(s_{\ell}\left(\left|A_{12}\right|^{2 r}\right)\right) \\
\leq & f\left(\sec ^{2 r}(\alpha) s_{\ell}^{\frac{r}{2}}\left(\left|A_{11}\right|\right) s_{\ell}^{\frac{r}{2}}\left(\left|A_{22}\right|\right)\right) \quad(\text { by }(12)) \\
= & f\left(\sec ^{r}(\alpha) s_{\ell}^{\frac{r}{2}}\left(\left|A_{11}\right|\right)\right) f\left(\sec ^{r}(\alpha) s_{\ell}^{\frac{r}{2}}\left(\left|A_{22}\right|\right)\right) \\
& (\text { since } f \text { is submultiplicative) }
\end{aligned}
$$


for $l=1,2, \ldots, n$. Let $\alpha=\left(\alpha_{1}, \alpha_{2}, \ldots, \alpha_{n}\right)$ be a decreasing sequence of nonnegative real numbers. The $\alpha$-norm of a matrix $B \in \mathbb{M}_{n}$ is defined by

$$
\|B\|_{\alpha}=\sum_{\ell=1}^{n} \alpha_{\ell} s_{\ell}(B)
$$

The $\alpha$-norms are unitarily invariant [4, p. 204].

Actually, inequality (13) means that

$$
\prod_{\ell=1}^{k} \alpha_{\ell} s_{\ell}\left(f\left(\left|A_{12}\right|^{2 r}\right)\right) \leq \prod_{\ell=1}^{k} \alpha_{\ell} s_{\ell}\left(f\left(\left(\sec ^{2}(\alpha)\left|A_{11}\right|\right)^{\frac{r}{2}}\right)\right) s_{\ell}\left(f\left(\left(\sec ^{2}(\alpha)\left|A_{22}\right|\right)^{\frac{r}{2}}\right)\right)
$$

for $k=1,2, \ldots, n$, which implies that

$$
\sum_{\ell=1}^{k} \alpha_{\ell} s_{\ell}\left(f\left(\left|A_{12}\right|^{2 r}\right)\right) \leq \sum_{\ell=1}^{k} \alpha_{\ell} s_{\ell}\left(f\left(\left(\sec ^{2}(\alpha)\left|A_{11}\right|\right)^{\frac{r}{2}}\right)\right) s_{\ell}\left(f\left(\left(\sec ^{2}(\alpha)\left|A_{22}\right|\right)^{\frac{r}{2}}\right)\right)
$$

for $k=1,2, \ldots, n$. Thus,

$$
\begin{aligned}
& \left\|f\left(\left|A_{12}\right|^{2 r}\right)\right\|_{\alpha} \\
& \quad=\sum_{\ell=1}^{n} \alpha_{\ell} s_{\ell}\left(f\left(\left|A_{12}\right|^{2 r}\right)\right) \\
& \quad \leq \sum_{\ell=1}^{n} \alpha_{\ell} s_{\ell}\left(f\left(\left(\sec ^{2}(\alpha)\left|A_{11}\right|\right)^{r / 2}\right)\right) s_{\ell}\left(f\left(\left(\sec ^{2}(\alpha)\left|A_{22}\right|\right)^{r / 2}\right)\right) \\
& \quad=\sum_{\ell=1}^{n} \alpha_{\ell}^{1 / s} s_{\ell}\left(f\left(\left(\sec ^{2}(\alpha)\left|A_{11}\right|\right)^{r / 2}\right)\right) \alpha_{\ell}^{1 / t} s_{\ell}\left(f\left(\left(\sec ^{2}(\alpha)\left|A_{22}\right|\right)^{r / 2}\right)\right) \\
& \quad \leq\left(\sum_{\ell=1}^{n} \alpha_{\ell} s_{\ell}^{s}\left(f\left(\left(\sec ^{2}(\alpha)\left|A_{11}\right|\right)^{r / 2}\right)\right)\right)^{1 / s}\left(\sum_{\ell=1}^{m} \alpha_{\ell} s_{\ell}^{t}\left(f\left(\left(\sec ^{2}(\alpha)\left|A_{22}\right|\right)^{r / 2}\right)\right)\right)^{1 / t}
\end{aligned}
$$

(by Hölder's inequality)

$$
\begin{aligned}
& =\left(\sum_{\ell=1}^{n} \alpha_{\ell} s_{\ell}\left(f^{s}\left(\left(\sec ^{2}(\alpha)\left|A_{11}\right|\right)^{r / 2}\right)\right)\right)^{1 / s}\left(\sum_{\ell=1}^{m} \alpha_{\ell} s_{\ell}\left(f^{t}\left(\left(\sec ^{2}(\alpha)\left|A_{22}\right|\right)^{r / 2}\right)\right)\right)^{1 / t} \\
& =\left\|f^{s}\left(\left(\sec ^{2}(\alpha)\left|A_{11}\right|\right)^{r / 2}\right)\right\|_{\alpha}^{1 / s}\left\|f^{t}\left(\left(\sec ^{2}(\alpha)\left|A_{22}\right|\right)^{r / 2}\right)\right\|_{\alpha}^{1 / t}
\end{aligned}
$$

for all decreasing sequences $\alpha=\left(\alpha_{1}, \ldots, \alpha_{n}\right)$ of nonnegative real numbers. It follows from the above inequality that

$$
\left\|f\left(\left|A_{12}\right|^{2 r}\right)\right\| \leq\left\|f^{s}\left(\left(\sec ^{2}(\alpha)\left|A_{11}\right|\right)^{r / 2}\right)\right\|^{1 / s}\left\|f^{t}\left(\left(\sec ^{2}(\alpha)\left|A_{22}\right|\right)^{r / 2}\right)\right\|^{1 / t} .
$$

The inequality for $A_{21}$ is similarly proved. 
Remark 1 In particular, when $A$ is a positive semidefinite contraction $(\alpha=0)$ and $r=1$, Theorem 2.4 gives

$$
\left\|f\left(\left|A_{12}\right|^{2}\right)\right\| \leq\left\|f^{s}\left(A_{11}^{1 / 2}\right)\right\|^{1 / s}\left\|f^{t}\left(A_{22}^{1 / 2}\right)\right\|^{1 / t},
$$

which is due to Bourahli et al. [1, Lemma 3.4]. Thus, our result (5) is a generalization of (14).

Remark 2 If $A$ is just a general sector matrix with $W(A) \subseteq S_{\alpha}$ for $\alpha \in\left[0, \frac{\pi}{2}\right.$ ) (not a contraction matrix), then we have the following result: Let $f \in \mathcal{C}$ be an increasing submultiplicative function on $[0, \infty)$ and $A=\left(\begin{array}{ll}A_{11} & A_{12} \\ A_{21} & A_{22}\end{array}\right) \in \mathbb{M}_{2 n}$ be a sector matrix with $W(A) \subseteq S_{\alpha}$ for some $\alpha \in[0, \pi / 2)$. Then, for all $r, s, t>0$ with $\frac{1}{s}+\frac{1}{t}=1$ and all unitarily invariant norms,

$$
\left\|f\left(\left|A_{12}\right|^{2 r}\right)\right\|=f\left(\sec ^{2 r}(\alpha)\left\|A_{11}\right\|_{\infty}^{\frac{r}{2}}\left\|A_{22}\right\|_{\infty}^{\frac{r}{2}}\right)\left\|f^{s}\left(\left|A_{11}\right|^{\frac{r}{2}}\right)\right\|^{\frac{1}{s}}\left\|f^{t}\left(\left|A_{22}\right|^{\frac{r}{2}}\right)\right\|^{\frac{1}{t}} .
$$

By (9), (10), and Lemma 2.1, we have

$$
\begin{aligned}
s_{\ell}\left(\left|A_{12}\right|^{r}\right) & =s_{\ell}^{r}\left(\left|A_{12}\right|\right)=s_{\ell}^{r}\left(A_{12}\right)=s_{\ell}^{r}\left(X_{1} Z X_{2}^{*}\right) \\
& \leq \sec ^{r}(\alpha)\left\|A_{11}\right\|_{\infty}^{\frac{r}{2}} s_{\ell}^{\frac{r}{2}}\left(\left|A_{22}\right|\right)
\end{aligned}
$$

and

$$
s_{\ell}\left(\left|A_{12}\right|^{r}\right) \leq \sec ^{r}(\alpha)\left\|A_{22}\right\|_{\infty}^{\frac{r}{2}} s_{\ell}^{\frac{r}{2}}\left(\left|A_{11}\right|\right)
$$

for $l=1,2, \ldots, n$.

Multiplying inequalities (16) and (17) by each other implies that

$$
s_{\ell}\left(\left|A_{12}\right|^{2 r}\right) \leq \sec ^{2 r}(\alpha)\left\|A_{11}\right\|_{\infty}^{\frac{r}{2}}\left\|A_{22}\right\|_{\infty}^{\frac{r}{2}} s_{\ell}^{\frac{r}{2}}\left(\left|A_{11}\right|\right) s_{\ell}^{\frac{r}{2}}\left(\left|A_{22}\right|\right)
$$

for $l=1,2, \ldots, n$.

So,

$$
\begin{aligned}
s_{\ell}\left(f\left(\left|A_{12}\right|^{2 r}\right)\right) & =f\left(s_{\ell}\left(\left|A_{12}\right|^{2 r}\right)\right) \\
& \leq f\left(\sec ^{2 r}(\alpha)\left\|A_{11}\right\|_{\infty}^{\frac{r}{2}}\left\|A_{22}\right\|_{\infty}^{\frac{r}{2}} s_{\ell}^{\frac{r}{2}}\left(\left|A_{11}\right|\right) s_{\ell}^{\frac{r}{2}}\left(\left|A_{22}\right|\right) \quad\right. \text { (by (18)) } \\
& =f\left(\sec ^{2 r}(\alpha)\left\|A_{11}\right\|_{\infty}^{\frac{r}{2}}\left\|A_{22}\right\|_{\infty}^{\frac{r}{2}}\right) f\left(s_{\ell}^{\frac{r}{2}}\left(\left|A_{11}\right|\right)\right) f\left(s_{\ell}^{\frac{r}{2}}\left(\left|A_{22}\right|\right)\right)
\end{aligned}
$$

for $l=1,2, \ldots, n$. Based on (19), we can obtain the desired result by a proof similar to that given for inequality (13). Therefore, when $\alpha=0$ and $r=1$, our result (15) is (2).

Theorem 2.5 Let $f \in \mathcal{C}$ be an increasing submultiplicative function on $[0, \infty)$ and $A \in \mathbb{M}_{2 n}$ be a contraction matrix partitioned as

$$
A=\left(\begin{array}{ll}
A_{11} & A_{12} \\
A_{21} & A_{22}
\end{array}\right),
$$


with $W(A) \subseteq S_{\alpha}$ for some $\alpha \in[0, \pi / 2)$. Then, for all $r, s, t>0$ with $\frac{1}{s}+\frac{1}{t}=1$ and all unitarily invariant norms,

$$
\begin{aligned}
& \left\|f\left(\left|A_{12}\right|^{2 r}\right)\right\|+\left\|f\left(\left|A_{21}\right|^{2 r}\right)\right\| \\
& \quad \leq 2\left\|f^{s}\left(\left(\sec ^{2}(\alpha)\left|A_{11}\right|\right)^{r / 2}\right)\right\|^{1 / s}\left\|f^{t}\left(\left(\sec ^{2}(\alpha)\left|A_{22}\right|\right)^{r / 2}\right)\right\|^{1 / t} .
\end{aligned}
$$

Proof By Theorem 2.4, we can have the desired result.

Remark 3 When $f(t)=t, r=1$, and $\alpha=\frac{\pi}{4}$, result (20) becomes

$$
\left\|\left|A_{12}\right|^{2}\right\|+\left\|\left|A_{21}\right|^{2}\right\| \leq 2\left\|f^{s}\left(\left(\sec ^{2}(\alpha)\left|A_{11}\right|\right)^{r / 2}\right)\right\|^{1 / s}\left\|f^{t}\left(\left(\sec ^{2}(\alpha)\left|A_{22}\right|\right)^{r / 2}\right)\right\|^{1 / t}
$$

\section{Acknowledgements}

The author wants to express her sincere thanks to the referee for his or her valuable remarks and suggestions, which made this paper more readable.

\section{Funding}

Zhou's work was supported by the High-level Talents Program of Natural Science Foundation of Hainan Province, China (2019RC193) and the Scientific Research Project of Colleges and Universities of Hainan Province, China (Hnky2020-31).

\section{Availability of data and materials}

Not applicable.

\section{Competing interests}

The author declares that they have no competing interests.

\section{Authors' contributions}

The author drafted and approved the final manuscript.

\section{Author details}

${ }^{1}$ Department of Mathematics and Statistics, Hainan Normal University, Haikou, China. ${ }^{2}$ Key Laboratory of Data Science and Intelligence Education Ministry of Education, Hainan Normal University, Haikou, China. ${ }^{3}$ Key Laboratory of Computational Science and Application of Hainan Province, Haikou, China.

\section{Publisher's Note}

Springer Nature remains neutral with regard to jurisdictional claims in published maps and institutional affiliations.

Received: 27 July 2020 Accepted: 12 November 2020 Published online: 19 November 2020

\section{References}

1. Bourahli, A., Hirzallah, O., Kittaneh, F.: Unitarily invariant norm inequalities for functions of accretive-dissipative $2 \times 2$ block matrices. Positivity (2020). https://doi.org/10.1007/s11117-020-00770-w

2. Bhatia, R.: Matrix Analysis. GTM, vol. 169. Springer, Berlin (1997)

3. Horn, R.A., Johnson, C.R.: Matrix Analysis, 2nd edn. Cambridge University Press, New York (2013)

4. Horn, R.A., Johnson, C.R.: Topics in Matrix Analysis. Cambridge University Press, Cambridge (1991)

5. Drury, S.W., Lin, M.: Singular value inequalities for matrices with numerical ranges in a sector. Oper. Matrices 8 , 1143-1148 (2014)

6. Fu, X., Liu, Y.: Rotfel'd inequality for partitioned matrices with numerical ranges in a sector. Linear Multilinear Algebra 64, 105-109 (2015)

7. Li, C.K., Sze, N.: Determinantal and eigenvalue inequalities for matrices with numerical ranges in a sector. J. Math. Anal. Appl. 410, 487-491 (2014)

8. Zhang, F.: A matrix decomposition and its applications. Linear Multilinear Algebra 63, 2033-2042 (2015)

9. Yang, J., Lu, L., Chen, Z.: Schatten $q$-norms and determinantal inequalities for matrices with numerical ranges in a sector. Linear Multilinear Algebra 67, 221-227 (2019)

10. Yang, J., Lu, L., Chen, Z:: A refinement of Rotfel'd type inequality for partitioned matrices with numerical ranges in a sector. Linear Multilinear Algebra 67, 1719-1726 (2019)

11. George, A., Ikramov, Kh.D.: On the properties of accretive-dissipative matrices. Math. Notes 77, 767-776 (2005)

12. Gumus, I.H., Hirzallah, O., Kittaneh, F.: Norm inequalities involving accretive-dissipative $2 \times 2$ block matrices. Linear Algebra Appl. 528, 76-93 (2017)

13. Jabbarzadeh, M.R., Kaleibary, V.: Inequalities for accretive-dissipative block matrices involving convex and concave functions Linear Multilinear Algebra (2020). https://doi.org/10.1080/03081087.2020.1726277

14. Kittaneh, F., Sakkijha, M.: Inequalities for accretive-dissipative matrices. Linear Multilinear Algebra 67, 1037-1042 (2019) 
15. Lin, M.: Reversed determinantal inequalities for accretive-dissipative matrices. Math. Inequal. Appl. 12, 955-958 (2012)

16. Lin, M., Zhou, D.: Norm inequalities for accretive-dissipative operator matrices. J. Math. Anal. Appl. 407, 436-442 (2013)

17. Lin, M.: Fischer type determinantal inequalities for accretive-dissipative matrices. Linear Algebra Appl. 438, 2808-2812 (2013)

18. Hiai, F.: Log-majorizations and norm inequalities for exponential operators. In: Linear Operators. Banach Center Publ., vol. 38, pp. 119-181 (1997)

19. Zhang, F:: Matrix Theory: Basic Results and Techniques, 2nd edn. Springer, New York (2011)

Submit your manuscript to a SpringerOpen ${ }^{\circ}$ journal and benefit from:

- Convenient online submission

- Rigorous peer review

Open access: articles freely available online

- High visibility within the field

- Retaining the copyright to your article

Submit your next manuscript at $\gg$ springeropen.com 